\title{
A Study of Approximation Properties in Felbin-Fuzzy Normed Spaces
}

\author{
Ju Myung Kim and Keun Young Lee * \\ Department of Mathematics and Statistics, Sejong University, Seoul 05006, Korea; kjm21@sejong.ac.kr \\ * Correspondence: bst21@sejong.ac.kr; Tel.: +82-2-6935-2504
}

Received: 23 December 2019; Accepted: 20 January 2020; Published: 23 January 2020

\begin{abstract}
In this paper, approximation properties in Felbin-fuzzy normed spaces are studied. These approximation properties have been recently introduced in Felbin-fuzzy normed spaces. We make topological tools to analyze such approximation properties. We especially develop the representation of dual spaces related to our contexts. By using this representation, we establish characterizations of approximation properties in terms of infinite sequences. Finally, we provide dual problems for approximation properties and their results in our contexts.
\end{abstract}

Keywords: Felbin-fuzzy normed space; approximation property; bounded approximation property; topological duals; dual problems

\section{Introduction}

Since Katsaras first introduced the notion of fuzzy norm on a vector space, a study for fuzzy normed spaces has been actively progressed [1]. In 1992, Felbin introduced a new definition of a fuzzy norm (namely, Felbin-fuzzy norm) related to a specific fuzzy metric [2,3]. In 2003, Bag and Samanta defined a more general notion of a fuzzy norm (namely, B-S fuzzy norm) [4,5]. Because of their pioneering research, topological properties have been studied according to Felbin type's fuzzy norms and B-S type's fuzzy norms, respectively [6-8]. Cho et al. investigated the classical and recent results of fuzzy normed spaces and fuzzy operators in their book [9].

The approximation property (AP) is an essential concept in researching functional analysis because the AP has been studied for the Shauder basis and operator theory. The AP means that the identity operator on an Banach space can be approximated in the compact open topology by finite rank operators (please see References [10-13]). In 2010 and 2016, Yilmaz et al. introduced the approximation property in B-S fuzzy normed spaces [14,15]. In 2019, the approximation property in Felbin fuzzy normed spaces was introduced [16]. Related works emerged from fuzzy theory. We would refer the reader to where intuitionistic fuzzy Banach space theory is outlined in Reference [17].

In this paper, we study approximation properties in Felbin-fuzzy normed spaces. Moreover, we will develop topological tools related approximation properties in Felbin-fuzzy normed spaces. We first identify approximation properties in Felbin-fuzzy normed spaces in terms of infinite sequences. We provide dual problems for such approximation properties. The advantage of our context is to characterize a compact subset in Felbin-fuzzy normed spaces.

Our paper is organized as follows-Section 2 is comprised of some preliminary results. In Section 3, we develop the representation of topological dual elements related approximation properties in Felbin-fuzzy normed spaces. Section 4 is devoted to identifying approximation properties in Felbin-fuzzy normed spaces in terms of infinite sequences. In Section 5, we apply this identification to provide some results about dual problems for approximation properties in Felbin-fuzzy normed spaces. 


\section{Preliminaries}

Definition 1 (See Reference [5]). A mapping $\eta: \mathbb{R} \rightarrow[0,1]$ is called a fuzzy real number with $\alpha$-level set $[\eta]_{\alpha}=\{t: \eta(t) \geqslant \alpha\}$, if it satisfies the following conditions:

(i) there exists a $t_{0} \in \mathbb{R}$ such that $\eta\left(t_{0}\right)=1$

(ii) for each $\alpha \in(0,1]$, there exist real numbers $\eta_{\alpha}^{-} \leqslant \eta_{\alpha}^{+}$such that the $\alpha$-level set $[\eta]_{\alpha}$ is equal to the closed interval $\left[\eta_{\alpha}^{-}, \eta_{\alpha}^{+}\right]$

The set of all fuzzy real numbers is denoted by $F(\mathbb{R})$. If $\eta \in F(\mathbb{R})$ and $\eta(t)=0$ whenever $t<0$, then $\eta$ is called a non-negative fuzzy real number and $F^{*}(\mathbb{R})$ denotes the set of all non-negative fuzzy real numbers. Define a partial ordering by $\eta \preceq \gamma$ in $F(\mathbb{R})$ if and only if $\eta_{\alpha}^{-} \leqslant \gamma_{\alpha}^{-}, \eta_{\alpha}^{+} \leqslant \gamma_{\alpha}^{+}$, for all $\alpha \in(0,1]$. Since each $r \in \mathbb{R}$ can be considered as the fuzzy real number $\tilde{r} \in F(\mathbb{R})$ denoted by

$$
\tilde{r}(t)= \begin{cases}1, & t=r \\ 0, & t \neq r,\end{cases}
$$

hence it follows that $\mathbb{R}$ can be embedded in $F(\mathbb{R})$ (See [5]).

Definition 2 (See Reference [5]). Let $X$ be a vector space over $\mathbb{R}$. Assume the mappings $L, R:[0,1] \times$ $[0,1] \rightarrow[0,1]$ are symmetric and non-decreasing in both arguments, and that $L(0,0)=0$ and $R(1,1)=1$. Let $\|\cdot\|: X \rightarrow F^{*}(\mathbb{R})$. The quadruple $(X,\|\cdot\|, L, R)$ is called a Felbin-fuzzy normed space with the fuzzy norm $\|\cdot\|$, if the following conditions are satisfied:

(F1) if $x \neq 0$, then $\inf _{0<\alpha \leqslant 1}\|x\|_{\alpha}^{-}>0$,

(F2) $\|x\|=\tilde{0}$ if and only if $x=0$,

(F3) $\|r x\|=|\tilde{r}|\|x\|$ for $x \in X$ and $r \in \mathbb{R}$,

(F4) for all $x, y \in X$,

(F4L) $\|x+y\|(s+t) \geqslant L(\|x\|(s),\|y\|(t))$ whenever $s \leqslant\|x\|_{1}^{-}, t \leqslant\|y\|^{-}$and $s+t \leqslant\|x+y\|_{1}^{-}$,

(F4R) $\|x+y\|(s+t) \leqslant R(\|x\|(s),\|y\|(t))$ whenever $s \geqslant\|x\|_{1}^{-}, t \geqslant\|y\|^{-}$and $s+t \geqslant\|x+y\|_{1}^{-}$.

The following definition gives the notion of strongly fuzzy bounded. Here, we use the arithmetic multiplicative operation $\otimes$ on $F(\mathbb{R}) \times F(\mathbb{R})$ as in [2]:

$$
(\eta \otimes \gamma)(t)=\sup _{t=x y}(\min (\eta(x), \gamma(y)))
$$

Definition 3 (See Reference [18]). Let $(X,\|\cdot\|)$ and $\left(Y,\|\cdot\|^{\sim}\right)$ be Felbin-fuzzy normed spaces. The linear operator $T: X \rightarrow Y$ is said to be a strongly fuzzy bounded if there is a real number $M>0$ such that $\|T x\| \sim \preceq \tilde{M} \otimes\|x\|$ for all $x \in X$. We will denote the set of all strongly fuzzy bounded operators from $(X,\|\cdot\|)$ to $\left(Y,\|\cdot\|^{\sim}\right)$ by $F(X, Y)$. Then $F(X, Y)$ is a vector space. For all $M>0$ we denote $F(X, Y, M)$ by

$$
\left\{T \in F(X, Y):\|T x\|^{\sim} \preceq \tilde{M} \otimes\|x\|, \forall x \in X, \forall t \in \mathbf{R}\right\}
$$

where $M$ is a positive real number.

$\mathcal{A}$ is called a bounded in $F(X, Y)$ if $\mathcal{A}=F(X, Y, M)$ for some $M>0$. Moreover, we denote the set of all finite rank strongly fuzzy bounded operators from $(X,\|\cdot\|)$ to $\left(Y,\|\cdot\|^{\sim}\right)$ by $\mathcal{F}(X, Y)$. Then $\mathcal{F}(X, Y)$ is a subspace of $F(X, Y)$. We similarly define $\mathcal{F}(X, Y, M)$ for some $M>0$. Now, we provide definitions of the approximation properties in Felbin-fuzzy normed spaces. For the definition and properties of $\alpha$-level set $(\alpha \in(0,1])$, see Reference [3,19].

Note that, if $Y=\mathbb{R}$, the linear space of all reals, we define a function $\|r\| \sim: \mathbb{R} \rightarrow[0,1]$ by

$$
\|r\|^{\sim}(t)= \begin{cases}1, & t=|r| \\ 0, & \text { otherwise. }\end{cases}
$$


Then $\|\cdot\| \sim$ is a fuzzy norm on $\mathbb{R}$ and $\alpha$-level sets of $\|r\|^{\sim}$ are given by $\left[\|r\|^{\sim}\right]_{\alpha}=[|r|,|r|]$ for all $0<\alpha \leqslant 1$.

Definition 4 (See Reference [20]). A strongly fuzzy bounded linear operator defined from a Felbin-fuzzy normed space $(X,\|\cdot\|)$ to $\left(\mathbb{R},\|\cdot\|^{\sim}\right)$ is called a strongly fuzzy bounded linear functional. We denote the set of all strongly fuzzy bounded linear functionals over $(X,\|\cdot\|)$ by $(X,\|\cdot\|)^{*}$. Define

$$
\|f\|_{\alpha}^{*-}=\sup _{x \in X, x \neq 0} \frac{|f(x)|}{\|x\|_{\alpha}^{+}},\|f\|_{\alpha}^{*+}=\sup _{x \in X, x \neq 0} \frac{|f(x)|}{\|x\|_{\alpha}^{-}}
$$

for all $f \in(X,\|\cdot\|)^{*}$.

Remark 1. Definition 4 came from Bag and Samanta [20]. Although they defined a strongly fuzzy bounded linear operator differently from this paper, the two definitions are same in the case of functionals.

Definition 5 (See Reference [21]). Let $(X,\|\cdot\|)$ be a Felbin-fuzzy normed space. A sequence $\left\{x_{n}\right\}$ of $X$ is said to converge to $x \in X\left(\lim _{n \rightarrow \infty} x_{n}=x\right)$ if $\lim _{n \rightarrow \infty}\left\|x_{n}-x\right\|_{\alpha}^{+}=0$ for all $\alpha \in(0,1]$. A subset $A$ of $X$ is called compact in $(X,\|\cdot\|)$ if each sequence of elements of $A$ has a convergent subsequence in $(X,\|\cdot\|)$.

Given a Felbin-fuzzy normed space $(X,\|\cdot\|)$, we recall

$$
B(x, \alpha, \varepsilon)=\left\{y \in X:\|x-y\|_{\alpha}^{+}<\varepsilon\right\} .
$$

Definition 6 (See Reference [21]). Let $(X,\|\cdot\|)$ be a Felbin-fuzzy normed space and $A \subseteq X$. A point $x_{0} \in X$ is called a closure of $A$ if $\left\{x_{0}+B(0, \alpha, \alpha)\right\} \cap A \neq \varnothing$. A sequence $\left\{x_{n}\right\}$ of $X$ is called a cauchy sequence if $\lim _{n, m \rightarrow \infty}\left\|x_{n}-x_{m}\right\|_{\alpha}^{+}=0$ for all $\alpha \in(0,1]$. A subset $A \subseteq X$ is said to be complete if every cauchy sequence in A converges in $A$.

Definition 7 (See Reference [16]). A Felbin-fuzzy normed space $(X,\|\cdot\|)$ is said to have the approximation property, briefly $A P$, if for every compact set $K$ in $(X,\|\cdot\|)$ and for each $\alpha \in(0,1]$ and $\varepsilon>0$, there exists an operator $T \in \mathcal{F}(X, X)$ such that

$$
\|T(x)-x\|_{\alpha}^{+} \leqslant \varepsilon
$$

for every $x \in K$.

As an example, having the AP in a Felbin-fuzzy normed space, let us consider Banach space $\ell_{\infty}$ with $\|x\|_{\infty}=\sup _{n}\left|x_{n}\right|$. Moreover, $\|x\|_{0}=\sup _{n}\left|\frac{x_{n}}{n}\right|$ is another norm on $\ell_{\infty}$. Now let us define

$$
\|x\|(t)= \begin{cases}1, & t=\|x\|_{0} \\ 1 / 2, & \|x\|_{0} \leqslant t<\|x\|_{\infty} \\ 0, & \text { otherwise }\end{cases}
$$

Then $\left(\ell_{\infty},\|\|\right)$ has the AP in a Felbin-fuzzy normed space ([16], Example 1). Moreover, in Reference [16], we made a comparison study among approximation properties in Felbin fuzzy normed spaces with other fuzzy normed spaces.

Definition 8 (See Reference [16]). Let $\lambda$ be a positive real number. A Felbin-fuzzy normed space $(X,\|\cdot\|)$ is said to have the $\lambda$-bounded approximation property, briefly $\lambda$-BAP, if for every compact set $K$ in $(X,\|\cdot\|)$ and for each $\alpha \in(0,1]$ and $\varepsilon>0$, there exists an operator $T \in \mathcal{F}(X, X, \lambda)$ such that

$$
\|T(x)-x\|_{\alpha}^{+} \leqslant \varepsilon
$$

for every $x \in K$. Also we say that $(X,\|\cdot\|)$ has the BAP if $(X,\|\cdot\|)$ has the $\lambda$-BAP for some $\lambda>0$. 


\section{The Dual Space of $(F(X, Y), \tau)$}

In this section, we give a representation of the dual space of $F(X, Y)$ endowed with the topology $\tau$ of uniform convergence on compact subsets of $(X,\|\cdot\|)$ where $(X,\|\cdot\|)$ and $\left(Y,\|\cdot\|^{\sim}\right)$ are Felbin-fuzzy normed spaces. We recall the following definition.

Definition 9 (See Reference [16]). Let $(X,\|\cdot\|)$ and $(Y,\|\cdot\| \sim)$ be Felbin-fuzzy normed spaces. For a compact $K \subset(X,\|\cdot\|), \varepsilon>0, \alpha \in(0,1]$, and $T \in F(X, Y)$ we put

$$
N e(T, K, \alpha, \varepsilon)=\left\{R \in F(X, Y): \sup _{x \in K}\|T x-R x\|_{\alpha}^{\sim^{+}}<\varepsilon\right\} .
$$

Let $\mathcal{S}$ be the collection of all such $\mathrm{Ne}(T, K, \alpha, \varepsilon)^{\prime}$ s. Then the $\tau$-topology on $F(X, Y)$ is the topology generated by $\mathcal{S}$. Clearly, $\tau$ is locally convex topology. Moreover, $(F(X, Y), \tau)^{*}$ is the vector spaces of all continuous fucntionals of $F(X, X)$

The $\tau$-topology is important tool to study approximation properties as followings. The proofs are trivial.

Theorem 1. Let $(X,\|\cdot\|)$ be a Felbin-fuzzy normed space and $I \in F(X, X)$ be an identity operator and $\lambda$ be a positive real number.

(a) $I \in \overline{\mathcal{F}(X, X)}^{\tau}$ if and only if $X$ has the $A P$.

(b) $I \in \overline{\mathcal{F}(X, X, \lambda)^{\tau}}$ if and only if $X$ has the $\lambda-B A P$.

Now we establish a representation of $(F(X, Y), \tau)^{*}$, which will be applied to characterize the approximation property and bounded approximation property in fuzzy normed spaces. The proof shall be given later.

Theorem 2. Let $(X,\|\cdot\|)$ and $\left(Y,\|\cdot\|^{\sim}\right)$ be complete Felbin-fuzzy normed spaces. Then $(F(X, Y), \tau)^{*}$ consists of all functionals $\varphi$ of the form

$$
\varphi(T)=\sum_{n=1}^{\infty} y_{n}^{*}\left(T x_{n}\right)
$$

where $\left(x_{n}\right) \subset(X,\|\cdot\|)$ and $\left(y_{n}^{*}\right) \subset\left(Y,\|\cdot\|_{\alpha_{1}}^{+}\right)^{*}$ for some $\left(\alpha_{n}\right) \subseteq(0,1] \downarrow 0$ such that

$$
\sum_{n=1}^{\infty}\left\|x_{n}\right\|_{\alpha_{n}}^{+}\left\|y_{n}^{*}\right\|_{\alpha_{1}}^{+}<\infty
$$

By the proof of (Reference [15], Lemma 4.2), we have the following.

Lemma 1. Let $(X,\|\cdot\|)$ be a Felbin-fuzzy normed space and $K$ be a compact subset in $(X,\|\cdot\|)$. Then there exists a finite set $\left\{x_{1}, x_{2}, \ldots, x_{n}\right\}$ in $K$ such that for $x \in K$, we have $x \in B\left(x_{i}, \alpha, \varepsilon\right)$ for some $x_{i}$.

The following Theorem shows that a relatively compact subset of a fuzzy normed space contained in the convex hull of a null sequence. Here, the convex hull of a subset $A$ of a topological vector space $X$, denoted by $\operatorname{co}(A)$, is the smallest convex set that includes $A$ and we denote the closure of $\operatorname{co}(A)$ by $\overline{c o}(A)$.

Theorem 3. Let $(X,\|\cdot\|)$ be a Felbin-fuzzy normed space. Suppose that $K$ is a relatively compact subset of $(X,\|\cdot\|)$. Then there is a sequence $\left(x_{n}\right)$ in $X$ converging to 0 in $(X,\|\cdot\|)$ such that

$$
K \subseteq \overline{c o}\left(\left\{x_{n}\right\}\right) .
$$


Proof. First, we recall that $a B(0, \alpha, \varepsilon)=B(0, \alpha, a \varepsilon)$ for each $\alpha \in(0,1)$ and $\varepsilon>0$ and $a>0$ and

$$
B\left(x, \alpha_{1}, \varepsilon_{1}\right) \subseteq B\left(x, \alpha_{2}, \varepsilon_{2}\right)
$$

whenever $x \in X, \alpha_{1}<\alpha_{2}$, and $0<\varepsilon_{1}<\varepsilon_{2}$ ([21], Lemma 3.1). It may be assumed that $K \neq \varnothing$. Since $2 K$ is relatively compact, ([21], Theorem 4.2$), 2 \mathrm{~K}$ is fuzzy bounded in $(X,\|\cdot\|)$. Then there exists $t\left(\frac{3}{4}\right)>0$ such that

$$
2 K \subseteq B(0,3 / 4, t(3 / 4))
$$

Also, by Lemma 1 , there exists a finite set $\left\{x_{1}, x_{2}, \ldots, x_{n_{1}}\right\}$ in $2 K$ such that $2 K \subseteq$ $\bigcup_{j=1}^{n_{1}} B\left(x_{j}, 2^{-1}, 2^{-1}\right)$. Let

$$
K_{1}=\bigcup_{j=1}^{n_{1}}\left(\left(2 K \cap B\left(x_{j}, 2^{-1}, 2^{-1}\right)\right)-x_{j}\right) .
$$

Then $K_{1} \subseteq B\left(0,2^{-1}, 2^{-1}\right)$. Since $K_{1}$ is nonempty and relatively compact, there exists a finite set $\left\{x_{n_{1}+1}, x_{n_{1}+2}, \ldots, x_{n_{2}}\right\}$ in $2 K_{1}$ such that $2 K_{1} \subseteq \bigcup_{j=n_{1}+1}^{n_{2}} B\left(x_{j}, 2^{-2}, 2^{-2}\right)$. Let

$$
K_{2}=\bigcup_{j=n_{1}+1}^{n_{2}}\left(\left(2 K_{1} \cap B\left(x_{j}, 2^{-2}, 2^{-2}\right)\right)-x_{j}\right)
$$

Then $K_{2} \subseteq B\left(0,2^{-2}, 2^{-2}\right)$. Since $K_{2}$ is nonempty and relatively compact, there exists a finite set $\left\{x_{n_{2}+1}, x_{n_{2}+2}, \ldots, x_{n_{3}}\right\}$ in $2 K_{2}$ such that $2 K_{2} \subseteq \bigcup_{j=n_{2}+1}^{n_{3}} B\left(x_{j}, 2^{-3}, 2^{-3}\right)$. Let

$$
K_{3}=\bigcup_{j=n_{2}+1}^{n_{3}}\left(\left(2 K_{2} \cap B\left(x_{j}, 2^{-3}, 2^{-3}\right)\right)-x_{j}\right)
$$

The construction of $\left(x_{n}\right)$ is continued in the obvious fashion.

Now, let $\alpha \in(0,1)$ and $\varepsilon>0$. Then we choose $k \in \mathbf{N}$ such that $2^{-k}<\alpha$ and $2^{-k+1}<\varepsilon$. By the above claim and $\left(x_{n}\right)^{\prime}$ s selection, we obtain that for each $\ell \geq k$

$$
\left(x_{j}\right)_{j=n_{\ell}+1}^{n_{\ell+1}} \subset 2 K_{\ell} \subset 2 B\left(0,2^{-\ell}, 2^{-\ell}\right)=B\left(0,2^{-\ell}, 2^{-\ell+1}\right) \subseteq B(0, \alpha, \varepsilon),
$$

hence we have $\left(x_{j}\right)_{j=n_{k}+1}^{\infty} \subset B(0, \alpha, \varepsilon)$. Thus, for all $j \geq n_{k}+1$, we have

$$
\left\|x_{j}\right\|_{\alpha}^{+}<\epsilon
$$

hence $\left(x_{n}\right)$ converges to 0 in $(X,\|\cdot\|)$.

Finally, $x_{0} \in K$. Since $2 x_{0} \in 2 K$, there is a positive integer $j_{1}$ such that $1 \leq j_{1} \leq n_{1}$ such that $2 x_{0}-x_{j_{1}} \in K_{1}$, so there is a positive integer $j_{2}$ such that $n_{1} \leq j_{2} \leq n_{2}$ such that $4 x_{0}-2 x_{j_{1}}-x_{j_{2}} \in K_{2}$, and so forth. If follows that for each $m \in \mathbf{N}$

$$
2^{m} x_{0}-2^{m-1} x_{j_{1}}-2^{m-2} x_{j_{2}} \cdots-x_{j_{m}} \in K_{m}
$$

Hence we have

$$
x_{0}-\sum_{n=1}^{m} 2^{-n} x_{j_{n}} \in 2^{-m} K_{m} \subseteq 2^{-m} B\left(0,2^{-m}, 2^{-m}\right)=B\left(0,2^{-m}, 2^{-2 m}\right) \subseteq B\left(0,2^{-m}, 2^{-m}\right)
$$

for each $m \in \mathbf{N}$, and therefore we have $x_{0}=\sum_{n} 2^{-n} x_{j_{n}} \in \overline{c o}\left(\left\{x_{n}\right\}\right)$. 
Corollary 1. Let $(X,\|\cdot\|)$ be a fuzzy normed space. Then there exists a sequence $\left(\alpha_{n}\right) \downarrow 0$ in $(0,1]$ such that

$$
\left\|x_{n}\right\|_{\alpha_{n}}^{+} \rightarrow 0
$$

where $\left(x_{n}\right)$ is the sequence selected in Theorem 3.

Proof. As we see in the proof of Theorem 3, we have a sequence $\left(n_{j}\right)_{j=0}^{\infty}$ in $\mathbf{N}$ with $n_{0}=0, n_{j} \uparrow \infty$ satisfying

$$
\begin{gathered}
x_{1}, x_{2}, \cdots, x_{n_{1}} \in 2 K \subseteq B(0,3 / 4, t(3 / 4)), \\
x_{n_{1}+1}, x_{n_{1}+2}, \cdots, x_{n_{2}} \in 2 K_{1} \subseteq B\left(0,2^{-1}, 1\right), \\
x_{n_{2}+1}, x_{n_{2}+2}, \cdots, x_{n_{3}} \in 2 K_{2} \subseteq B\left(0,2^{-2}, 2^{-1}\right), \\
\ldots \ldots \\
x_{n_{\ell}+1}, x_{n_{\ell}+2}, \cdots, x_{n_{\ell}} \in 2 K_{\ell} \subseteq B\left(0,2^{-\ell}, 2^{-\ell+1}\right),
\end{gathered}
$$

Then we obtain

$$
\begin{gathered}
\left\|x_{n}\right\|_{3 / 4} \leq t(3 / 4), \forall n=1,2, \cdots, n_{1}, \\
\left\|x_{n}\right\|_{\frac{1}{2}}^{+} \leq 1, \forall n=n_{1}+1, n_{1}+2, \cdots, n_{2}, \\
\left\|x_{n}\right\|_{\frac{1}{4}}^{+} \leq \frac{1}{2}, \forall n=n_{2}+1, n_{2}+2, \cdots, n_{3}, \\
\ldots \ldots \\
\left\|x_{n}\right\|_{\frac{1}{2^{\ell}}}^{+} \leq \frac{1}{2^{\ell-1}}, \forall n=n_{\ell}+1, n_{\ell}+2, \cdots, n_{\ell+1},
\end{gathered}
$$

Now we put

$$
\alpha_{n}= \begin{cases}\frac{3}{4}, & 1 \leqslant n \leqslant n_{1} \\ \frac{1}{2}, & n_{1}+1 \leqslant n \leqslant n_{2} \\ \frac{1}{2^{2}}, & n_{2}+1 \leqslant n \leqslant n_{3} \\ \cdots & \\ \frac{1}{2^{\ell}}, & n_{\ell}+1 \leqslant n \leqslant n_{\ell+1} . \\ \cdots & \end{cases}
$$

Clearly, we have $\left(\alpha_{n}\right) \downarrow 0$ in $(0,1]$ and

$$
\left\|x_{n}\right\|_{\alpha_{n}}^{+} \rightarrow 0
$$

The following lemma can be obtained from the proof of (Reference [15], Lemma 5.7).

Lemma 2. Let $(X,\|\cdot\|)$ and $\left(Y,\|\cdot\|^{\sim}\right)$ be Felbin-fuzzy normed spaces. If $\varphi \in(F(X, Y), \tau)^{*}$, then there exists a finite subset $\left\{x_{1}, x_{2}, \ldots, x_{n}\right\}$ of $X$ and $\alpha \in(0,1]$ and $\varepsilon>0$ such that $T \in \bigcap_{i=1}^{n}\left\{T \in F(X, Y):\left\|T x_{i}\right\|_{\alpha}^{2}<\right.$ $\varepsilon\}$ implies $|\varphi(T)|<1$.

Proof of Theorem 2. If a linear functional $\varphi$ on $F(X, Y)$ is given by

$$
\varphi(T)=\sum_{n=1}^{\infty} y_{n}^{*}\left(T x_{n}\right)
$$


where $\left(x_{n}\right) \subset(X,\|\cdot\|)$ and $\left(y_{n}^{*}\right) \subset\left(Y,\|\cdot\|_{\alpha_{1}^{+}}^{+}\right)^{*}$ for some $\left(\alpha_{n}\right) \subseteq(0,1] \downarrow 0$ such that

$$
\sum_{n=1}^{\infty}\left\|x_{n}\right\|_{\alpha_{n}}^{+}\left\|y_{n}^{*}\right\|_{\alpha_{1}}^{+}<\infty
$$

then we shall show that there are a compact subset $K$ of $(X,\|\cdot\|)$ and $B>0$ such that

$$
\varphi(T) \leq B
$$

for all $T \in N e\left(0, K, \alpha_{1}, 1\right)$, which proves that $\varphi$ is in $(F(X, Y), \tau)^{*}$. First, let $\left(\eta_{n}\right)$ be a sequence of positive reals so that

$$
\eta_{n} \uparrow \infty, \sum_{n=1}^{\infty} \eta_{n}\left\|x_{n}\right\|_{\alpha_{n}}^{+}\left\|y_{n}^{*}\right\|_{\alpha_{1}}^{+}<\infty .
$$

Put

$$
K=\{0\} \bigcup\left\{\frac{x_{n}}{\eta_{n}\left\|x_{n}\right\|_{\alpha_{n}}^{+}}\right\}
$$

and $B=\sum_{n=1}^{\infty} \eta_{n}\left\|x_{n}\right\|_{\alpha_{n}}^{+}\left\|y_{n}^{*}\right\|_{\alpha_{1}}^{+}$.

Now, we shall show that $K$ is a compact subset of $(X,\|\cdot\|)$. We are enough to prove that $\forall \beta \in(0,1]$,

$$
\left\|\frac{x_{n}}{\eta_{n}\left\|x_{n}\right\|_{\alpha_{n}}^{+}}\right\|_{\beta}^{+} \rightarrow 0
$$

Take any $\alpha_{1}<\beta \leq 1$. Since $\forall n \in \mathbf{N},\left\|x_{n}\right\|_{\beta}^{+} \leq\left\|x_{n}\right\|_{\alpha_{n}}^{+}$, we have $n \in \mathbf{N}$,

$$
\frac{\left\|x_{n}\right\|_{\beta}^{+}}{\eta_{n}\left\|x_{n}\right\|_{\alpha_{n}}^{+}} \leq \frac{1}{\eta_{n}}
$$

hence

$$
\left\|\frac{x_{n}}{\eta_{n}\left\|x_{n}\right\|_{\alpha_{n}}^{+}}\right\|_{\beta}^{+} \rightarrow 0
$$

Now we take any $\alpha_{2}<\beta \leq \alpha_{1}$. By the above argument, for $n \geq 2$,

$$
\frac{\left\|x_{n}\right\|_{\beta}^{+}}{\eta_{n}\left\|x_{n}\right\|_{\alpha_{n}}^{+}} \leq \frac{1}{\eta_{n}},
$$

hence we have

$$
\left\|\frac{x_{n}}{\eta_{n}\left\|x_{n}\right\|_{\alpha_{n}}^{+}}\right\|_{\beta}^{+} \rightarrow 0
$$

By continuing this process for $\beta$, we prove our claim. On the other hand, we have for all $T \in \mathrm{Ne}(0, K, \alpha, 1)$

$$
\begin{aligned}
|\varphi(T)| & \leq \sum_{n=1}^{\infty}\left|y_{n}^{*}\left(T x_{n}\right)\right|=\sum_{n=1}^{\infty} \mid y_{n}^{*}\left(T\left(\frac{x_{n}}{\eta_{n}\left\|x_{n}\right\|_{\alpha_{n}}^{+}}\right) \eta_{n}\left\|x_{n}\right\|_{\alpha_{n}}^{+} \mid\right. \\
& \leq \sup _{n}\left\|T\left(\frac{x_{n}}{\eta_{n}\left\|x_{n}\right\|_{\alpha_{1}}^{+}}\right)\right\|_{\alpha_{1}}^{\sim_{1}} \sum_{n=1}^{\infty} \eta_{n}\left\|x_{n}\right\|_{\alpha_{n}}^{+}\left\|y_{n}^{*}\right\|_{\alpha_{1}}^{+} \\
& \leq \sup _{x \in K}\|T(x)\|_{\alpha_{n}}^{\sim_{n}} \sum_{n=1}^{\infty} \eta_{n}\left\|x_{n}\right\|_{\alpha_{n}}^{+}\left\|y_{n}^{*}\right\|_{\alpha_{1}}^{+} \\
& \leq B .
\end{aligned}
$$


Now, for the converse, assume that $\varphi \in(F(X, Y), \tau)^{*}$. Then, by Lemma 2, there are compact sets $K_{1}, \ldots, K_{m}$ and $\alpha \in(0,1]$ and $\varepsilon>0$ such that $\sup _{x \in K_{i}}\|T x\|_{\alpha}^{+}<\varepsilon, T \in F(X, Y), i=1,2, \ldots, n$ implies $|\varphi(T)|<1$. Put $K=\bigcup_{i=1}^{n} K_{i}$ and $B>1 / \varepsilon$. Then it is easy to check that

$$
|\varphi(T)| \leq B \sup _{x \in K}\|T x\|_{\alpha}^{\sim+}
$$

for all $T \in F(X, Y)$. By Theorem 3, there is a sequence $\left(x_{n}\right)$ in $X$ converging to 0 in $(X,\|\cdot\|)$ such that

$$
K \subseteq \overline{C O}\left(\left\{x_{n}\right\}\right)
$$

Moreover, Corollary 1 , there exists a sequence $\left(\alpha_{n}\right) \downarrow 0$ in $(0,1]$ such that

$$
\left\|x_{n}\right\|_{\alpha_{n}}^{+} \rightarrow 0
$$

There exists $n_{0} \in \mathbf{N}$ such that $\alpha_{n_{0}} \leq \alpha$. Put

$$
\alpha_{n}^{\prime}= \begin{cases}\alpha, & 1 \leq n \leq n_{0} \\ \alpha_{n}, & n>n_{0}\end{cases}
$$

Then we may assume $\left(\alpha_{n}\right) \downarrow 0$ such that for all $n, \alpha_{n} \leq \alpha$ and

$$
\left\|x_{n}\right\|_{\alpha_{n}}^{+} \rightarrow 0
$$

Also, we can observe

$$
|\varphi(T)| \leq B \sup _{n}\left\|T x_{n}\right\|_{\alpha_{1}}^{\sim+}
$$

for all $T \in F(X, Y)$.

Now we put $Y_{1}=\left(Y,\|\cdot\|_{\alpha_{1}}^{+}\right)$Let us consider the linear map

$$
\begin{gathered}
\phi: F(X, Y) \rightarrow c_{0}\left(Y_{1}\right) \\
T \longrightarrow\left(T x_{n}\right) .
\end{gathered}
$$

Since for each $T \in F(X, Y)$, there exists $M>0$ such that

$$
\left\|T\left(x_{n}\right)\right\|_{\alpha_{1}}^{\sim^{+}} \leq M\left\|x_{n}\right\|_{\alpha_{1}}^{+} \leq M\left\|x_{n}\right\|_{\alpha_{n}}^{+}
$$

the linear map $\phi$ is well defined. Now we define $\Phi(\eta)=\varphi(T)$ if $\eta \in \phi(F(X, Y))$ and $\eta=\phi(T)$. We claim that $\Phi$ is well-defined and linear continuous functional on $\phi(F(X, Y))$. Indeed, observe that $\phi(T)=0$ implies $\sup _{n}\left\|T x_{n}\right\|_{\alpha_{n}}^{+}=0$, hence $\varphi(T)=0$. Thus $\Phi$ is well-defined. Also, we obtain that

$$
|\Phi(\eta)|=|\varphi(T)| \leq B \sup _{n}\left\|T x_{n}\right\|_{\alpha_{1}^{+}}^{+}=B\|\eta\|_{c_{0}\left(Y_{1}\right)}
$$

it proves our claim.

Now, by applying Hahn-Banach extension theorem, the functional $\Phi$ can be extended to a $\widehat{\Phi} \in c_{0}\left(Y_{1}\right)^{*}$. Then there is uniquely the sequence $\left(y_{n}^{*}\right)$ in $Y_{1}^{*}$ such that $\sum_{n=1}^{\infty}\left\|y_{n}^{*}\right\|_{\alpha_{1}}^{+}=\|\Phi\|$ and

$$
\Phi\left(\left(y_{n}\right)\right)=\sum_{n=1}^{\infty} y_{n}^{*}\left(y_{n}\right), \forall\left(y_{n}\right) \in c_{0}\left(Y_{1}\right)
$$

Since

$$
\left\|x_{n}\right\|_{\alpha_{n}}^{+} \leq 1
$$


we have

$$
\sum_{n=1}^{\infty}\left\|x_{n}\right\|_{\alpha_{n}}^{+}\left\|y_{n}^{*}\right\|_{\alpha_{1}}^{+}<\infty
$$

Thus, for each $T \in F(X, Y)$ we have

$$
\varphi(T)=\Phi\left(\left(T x_{n}\right)\right)=\widehat{\Phi}\left(\left(T x_{n}\right)\right)=\sum_{n=1}^{\infty} y_{n}^{*}\left(T x_{n}\right) .
$$

\section{Characterizations of Approximation Properties}

In this section, we establish new characterizations of approximation properties in Felbin-fuzzy normed spaces in terms of infinite sequences by applying the representation of Section 5 . The following includes our main theorems. The proof shall be given later.

Theorem 4. A complete Felbin-fuzzy normed space $(X,\|\cdot\|)$ has the AP if and only if for every $\left(\alpha_{n}\right) \subseteq(0,1] \downarrow$ $0,\left(x_{n}\right) \subset(X,\|\cdot\|)$ and $\left(x_{n}^{*}\right) \subset\left(X,\|\cdot\|_{\alpha_{1}}^{+}\right)^{*}$ such that

$$
\begin{aligned}
& \sum_{n=1}^{\infty}\left\|x_{n}\right\|_{\alpha_{n}}^{+}\left\|x_{n}^{*}\right\|_{\alpha_{1}}^{+}<\infty, \\
& \sum_{n=1}^{\infty} x_{n}^{*}(x) x_{n}=0, \forall x \in X,
\end{aligned}
$$

we have

$$
\sum_{n}^{\infty} x_{n}^{*}\left(x_{n}\right)=0
$$

Theorem 5. A complete Felbin-fuzzy normed space $(X,\|\cdot\|)$ has the BAP if and only if for every $\left(\alpha_{n}\right) \subseteq$ $(0,1] \downarrow 0,\left(x_{n}\right) \subset(X,\|\cdot\|)$ and $\left(x_{n}^{*}\right) \subset\left(X,\|\cdot\|_{\alpha_{n}}^{+}\right)^{*}$ such that

$$
\begin{aligned}
& \sum_{n=1}^{\infty}\left\|x_{n}\right\|_{\alpha_{n}}^{+}\left\|x_{n}^{*}\right\|_{\alpha_{1}}^{+}<\infty, \\
& \sum_{n=1}^{\infty} x_{n}^{*}(x) x_{n} \leq 1, \forall x \in X,
\end{aligned}
$$

we have

$$
\sum_{n}^{\infty} x_{n}^{*}\left(x_{n}\right) \leq 1
$$

Remark 2. Theorems 4, 5 are very important tools to solve dual problems for approximation properties in Felbin-fuzzy normed spaces (see Section 5).

Lemma 3. Let $(X,\|\cdot\|)$ be a Felbin-fuzzy normed space. Suppose that $\mathcal{C}$ is a subspace of $F(X, X)$. Let $T \in$ $F(X, X)$. Then the following are equivalent.
(a) $T$ belongs to $\overline{\mathcal{C}}^{\tau}$.
(b) For every $f \in(F(X, X), \tau)^{*}$ such that $f(S)=0$ for all $S \in \mathcal{C}$, we have $f(T)=0$.

Proof. This can be directly derived from a result of the locally convex space version of the Hahn-Banach theorem. One may refer to Reference [22], Corollary 2.2.20.

Moreover, we need the following lemma [23]. 
Lemma 4. Let $(X,\|\cdot\|)$ be a Felbin-fuzzy normed space. Suppose that $\mathcal{C}$ is a balanced convex subset of $F(X, X)$. Let $T \in F(X, X)$. Then the following are equivalent.

(a) $T$ belongs to $\overline{\mathcal{C}}^{\tau}$.

(b) For every $f \in(F(X, X), \tau)^{*}$ such that $|f(S)| \leq 1$ for all $S \in \mathcal{C}$, we have $|f(T)| \leq 1$.

Proposition 1. Let $Y$ be a finite dimensional subspace of a Felbin-fuzzy normed space $(X,\|\cdot\|)$. Suppose that $x \in X \backslash Y$. Then there is a strongly fuzzy bounded linear functional $f$ on $X$ such that $\sup _{\alpha \in(0,1]}\|f\|_{\alpha}^{*+}=1$, $f(x)=\inf _{y \in Y} \inf _{\alpha \in(0,1]}\|x-y\|_{\alpha}^{-}$and $Y \subseteq \operatorname{ker}(f)$.

Proof. The proof comes from Reference [16], Theorem 2.

The following theorem gives the representation of finite rank strongly fuzzy bounded operators ([16], Corollary 2).

Theorem 6. Let $(X,\|\cdot\|)$ and $\left(Y,\|\cdot\|^{\sim}\right)$ be Felbin-fuzzy normed spaces. If $T: X \rightarrow Y$ is a finite rank strongly fuzzy bounded linear operator, then there exist $\left(y_{n}\right)_{n=1}^{k} \subset Y$ and $\left(f_{n}\right)_{n=1}^{k} \subset(X,\|\cdot\|)^{*}$ such that

$$
T(x)=\sum_{n=1}^{k} f_{n}(x) y_{n}
$$

for all $x \in X$.

Now, we are ready to prove Theorems 4 and 5 .

Proof of Theorem 4. Let $\left(\alpha_{n}\right) \subseteq(0,1] \downarrow 0,\left(x_{n}\right) \subset(X,\|\cdot\|)$ and $\left(x_{n}^{*}\right) \subset\left(X,\|\cdot\|_{\alpha_{1}}^{+}\right)^{*}$ such that

$$
\begin{aligned}
& \sum_{n=1}^{\infty}\left\|x_{n}\right\|_{\alpha_{n}}^{+}\left\|x_{n}^{*}\right\|_{\alpha_{1}}^{+}<\infty, \\
& \sum_{n=1}^{\infty} x_{n}^{*}(x) x_{n}=0, \forall x \in X .
\end{aligned}
$$

Now a linear functional $\varphi$ on $F(X, X)$ is given by

$$
\varphi(T)=\sum_{n=1}^{\infty} x_{n}^{*}\left(T x_{n}\right) .
$$

By Theorem 2, we have $\varphi \in(F(X, X), \tau)^{*}$. Since $(X,\|\cdot\|)$ has the AP, we have

$$
I \in \overline{\mathcal{F}(X, X)}^{\tau} .
$$

Then, by Lemma 3, we are enough to show that

$$
\varphi(S)=0, \forall S \in \mathcal{F}(X, X) .
$$

We can observe that for each $x \in X$ and $x^{*} \in(X,\|\cdot\|)^{*}$, by assumption, we have

$$
x^{*}(0)=x^{*}\left(\sum_{n=1}^{\infty} x_{n}^{*}(x) x_{n}\right)=\sum_{n=1}^{\infty} x_{n}^{*}(x) x^{*}\left(x_{n}\right)=0,
$$


because $x^{*}$ is sequentially fuzzy continuous. Let $S$ be in $\mathcal{F}(X, X)$. By Theorem 6 , there exist $\left(z_{i}\right)_{i=1}^{k} \subset X$ and $\left(z_{i}^{*}\right)_{i=1}^{k} \subset(X,\|\cdot\|)^{*}$ such that

$$
S(x)=\sum_{i=1}^{k} z_{i}^{*}(x) z_{i}, \forall x \in X
$$

Then, by the above observation, we have

$$
\varphi(S)=\sum_{n=1}^{\infty} \sum_{i=1}^{k} x_{n}^{*}\left(z_{i}^{*}\left(x_{n}\right) z_{i}\right)=\sum_{i=1}^{k} \sum_{n=1}^{\infty} z_{i}^{*}\left(x_{n}\right) x_{n}^{*}\left(z_{i}\right)=0 .
$$

Conversely, let $\varphi$ be in $(F(X, X), \tau)^{*}$ and

$$
\varphi(S)=0, \forall S \in \mathcal{F}(X, X)
$$

Then, by Theorem 2, we have

$$
\varphi(T)=\sum_{n=1}^{\infty} x_{n}^{*}\left(T x_{n}\right)
$$

where $\left(x_{n}\right) \subset(X,\|\cdot\|)$ and $\left(x_{n}^{*}\right) \subset\left(X\|\cdot\|_{\alpha_{1}}^{+}\right)^{*}$ for some $\left(\alpha_{n}\right) \subseteq(0,1] \downarrow 0$ such that

$$
\sum_{n=1}^{\infty}\left\|x_{n}\right\|_{\alpha_{n}}^{+}\left\|x_{n}^{*}\right\|_{\alpha_{1}}^{+}<\infty
$$

By Lemma 3 and the assumption, we are enough to show that

$$
\varphi(I)=0 \text {, i.e. } \sum_{n=1}^{\infty} x_{n}^{*}(x) x_{n}=0, \forall x \in X .
$$

First, we claim that for each $x \in X, \sum_{n=1}^{\infty} x_{n}^{*}(x) x_{n}$ exists in $(X,\|\cdot\|)$. Indeed, take any $\alpha \in(0,1]$ and $\epsilon>0$ and $x \in X$. There is $k_{0} \in \mathbf{N}$ with $\alpha_{k_{0}} \leq \alpha$ such that for all $m>k_{0}$,

$$
\sum_{n=k_{0}}^{m}\|x\|_{\alpha_{1}}^{+}\left\|x_{n}\right\|_{\alpha_{n}}^{+}\left\|x_{n}^{*}\right\|_{\alpha_{1}}^{+}<\epsilon
$$

Then we have

$$
\left\|\sum_{n=k_{0}}^{m} x_{n}^{*}(x) x_{n}\right\|_{\alpha}^{+} \leq \sum_{n=k_{0}}^{m}\left|x_{n}^{*}(x)\right|\left\|x_{n}\right\|_{\alpha}^{+} \leq \sum_{n=k_{0}}^{m}\|x\|_{\alpha_{1}}^{+}\left\|x_{n}\right\|_{\alpha_{n}}^{+}\left\|x_{n}^{*}\right\|_{\alpha_{1}}^{+}<\epsilon,
$$

so $\left(\sum_{n=1}^{m} x_{n}^{*}(x) x_{n}\right)$ is Cauchy sequence. Since $(X,\|\cdot\|)$ is complete, $\sum_{n=1}^{\infty} x_{n}^{*}(x) x_{n}$ exists. Also, we claim that for each $x^{*} \in(X,\|\cdot\|)^{*}$ and $x \in X$, we have

$$
x^{*}\left(\sum_{n=1}^{\infty} x_{n}^{*}(x) x_{n}\right)=0
$$

Indeed, let $x^{*} \in(X,\|\cdot\|)^{*}$ and $x \in X$. Put

$$
S(z):=x^{*}(z) x, \forall z \in X .
$$

Then $S \in \mathcal{F}(X, X)$, by assumption, we have

$$
\varphi(S)=\sum_{n=1}^{\infty} x_{n}^{*}(x) x^{*}\left(x_{n}\right)=0 .
$$


Since $x^{*}$ is sequentially fuzzy continuous, we have

$$
x^{*}\left(\sum_{n=1}^{\infty} x_{n}^{*}(x) x_{n}\right)=\sum_{n=1}^{\infty} x_{n}^{*}(x) x^{*}\left(x_{n}\right)=0
$$

It follows that

$$
\sum_{n=1}^{\infty} x_{n}^{*}(x) x_{n}=0
$$

because if not, that is, $\sum_{n=1}^{\infty} x_{n}^{*}(x) x_{n} \neq 0$, then, by Proposition 1 , there exists $x^{*} \in(X,\|\cdot\|)^{*}$ such that

$$
x^{*}\left(\sum_{n=1}^{\infty} x_{n}^{*}(x) x_{n}\right)=\inf _{\alpha \in(0,1]}\left\|\sum_{n=1}^{\infty} x_{n}^{*}(x) x_{n}\right\|_{\alpha}^{-} \neq 0,
$$

it is a contradiction.

Proof of Theorem 5. Since $\mathcal{F}(X, X, \lambda)$ is a balanced convex, by Lemma 4 and similar arguments of Theorem 4, we can prove it.

\section{Application to the Dual Problems}

In this section, we provide dual problems for approximation properties in Felbin-fuzzy normed spaces and their partial solutions.

Dual problems. Let $(X,\|\cdot\|)$ be a Felbin-fuzzy normed space. If $(X,\|\cdot\|)^{*}$ has the AP, then does $(X,\|\cdot\|)$ have the AP? Conversely, if $(X,\|\cdot\|)$ has the AP, then does $(X,\|\cdot\|)^{*}$ have the AP?

In Banach space theory, dual problems were completely solved (see Reference [13]). However, in fuzzy normed spaces, we have partial solutions.

Proposition 2. Let $(X,\|\cdot\|)$ be a fuzzy normed space and $\alpha \in(0,1]$. We define

$$
\|x\|_{(\alpha)}(t)= \begin{cases}1, & t=\|x\|_{\alpha}^{+} \\ 0, & \text { otherwise. }\end{cases}
$$

Then $\left(X,\|\cdot\|_{(\alpha)}\right)$ is a fuzzy normed space. Also, for all $\beta \in(0,1]$ we have

$$
\left[\|x\|_{(\alpha)}\right]_{\beta}=\left[\|x\|_{\alpha}^{+},\|x\|_{\alpha}^{+}\right] .
$$

Proof. The proof is clear.

We have a partial solution for dual problems by giving the specific condition of a dual space as the following.

Theorem 7. Let $(X,\|\cdot\|)$ be a complete Felbin-fuzzy normed space. If for every $\alpha \in(0,1],\left(X,\|\cdot\|_{(\alpha)}\right)^{*}$ has the AP, then $(X,\|\cdot\|)$ has the AP.

Proof. We shall use Theorem 4. Let $\left(\alpha_{n}\right) \subseteq(0,1] \downarrow 0$. Also we fix $\left(x_{n}\right) \subset(X,\|\cdot\|)$ and $\left(x_{n}^{*}\right) \subset$ $\left(X,\|\cdot\|_{\alpha_{1}}^{+}\right)^{*}$ such that

$$
\begin{aligned}
& \sum_{n=1}^{\infty}\left\|x_{n}\right\|_{\alpha_{n}}^{+}\left\|x_{n}^{*}\right\|_{\alpha_{1}}^{+}<\infty, \\
& \sum_{n=1}^{\infty} x_{n}^{*}(x) x_{n}=0, \forall x \in X .
\end{aligned}
$$


Now let us consider $\left(X,\|\cdot\|_{\left(\alpha_{1}\right)}\right)$. Since, $\forall x \in X, \sum_{n=1}^{\infty} x_{n}^{*}(x) x_{n}$ converges to 0 in $\left(X,\|\cdot\|_{\left(\alpha_{1}\right)}\right)$, we have

$$
\sum_{n=1}^{\infty} x^{*}\left(x_{n}\right) x_{n}^{*}=0, \forall x^{*} \in\left(X,\|\cdot\|_{\left(\alpha_{1}\right)}\right)^{*} .
$$

We can observe that $\left(x_{n}^{*}\right) \subset\left(X,\|\cdot\|_{\left(\alpha_{1}\right)}\right)^{*}$ because for each $\alpha \in(0,1]$, by Proposition 2 , we have

$$
\left|x_{n}^{*}(x)\right| \leq M\|x\|_{\alpha_{1}}^{+}=M\|x\|_{\left(\alpha_{1}\right) \alpha}, \forall x \in X .
$$

Also, for each $x^{*} \in\left(X,\|\cdot\|_{\left(\alpha_{1}\right)}\right)^{*}$, by again Proposition 2, we obtain

$$
\left\|x^{*}\right\|_{\left(\alpha_{1}\right) \alpha}^{*+}=\sup \frac{\left|x^{*}(x)\right|}{\|x\|_{\left(\alpha_{1}\right) \alpha}^{-}}=\sup \frac{\left|x^{*}(x)\right|}{\|x\|_{\alpha_{1}}^{+}}=\left\|x^{*}\right\|_{\alpha_{1}}^{+},
$$

Now we define that for each $x \in X$,

$$
J(x)\left(x^{*}\right):=x^{*}(x), \forall x^{*} \in\left(X,\|\cdot\|_{\left(\alpha_{1}\right)}\right)^{*} .
$$

Then, $\forall x \in X$, we can regard $J(x)$ as the element of $\left(\left(X,\|\cdot\|_{\left(\alpha_{n}\right)}\right)^{*},\|\cdot\|_{\left(\alpha_{n}\right) \alpha_{1}}^{*+}\right)^{*}$ because

$$
\|J(x)\|_{\left(\alpha_{1}\right) \alpha_{1}}^{+}=\sup \frac{\left|J(x)\left(x^{*}\right)\right|}{\left\|x^{*}\right\|_{\left(\alpha_{1}\right) \alpha_{1}}^{*+}}=\sup \frac{\left|x^{*}(x)\right|}{\left\|x^{*}\right\|_{\left(\alpha_{1}\right) \alpha_{1}}^{*+}}=\sup \frac{\left|x^{*}(x)\right|}{\left\|x^{*}\right\|_{\alpha_{1}}^{+}}=\|x\|_{\alpha_{1}}^{+} .
$$

Thus it follows $\left(J\left(x_{n}\right)\right) \subset\left(\left(X,\|\cdot\|_{\left(\alpha_{1}\right)}\right)^{*},\|\cdot\|_{\left(\alpha_{1}\right) \alpha_{1}}^{*+}\right)^{*}$, hence we have

$$
\sum_{n=1}^{\infty}\left\|x_{n}^{*}\right\|_{\left(\alpha_{1}\right) \alpha_{n}}^{*+}\left\|J\left(x_{n}\right)\right\|_{\left(\alpha_{1}\right) \alpha_{1}}^{+} \leq \sum_{n=1}^{\infty}\left\|x_{n}\right\|_{\alpha_{1}}^{+}\left\|x_{n}^{*}\right\|_{\alpha_{1}}^{+} \leq \sum_{n=1}^{\infty}\left\|x_{n}\right\|_{\alpha_{n}}^{+}\left\|x_{n}^{*}\right\|_{\alpha_{1}}^{+}<\infty
$$

Then we have

$$
\sum_{n=1}^{\infty} J\left(x_{n}\right)\left(x^{*}\right) x_{n}^{*}=0, \forall x^{*} \in\left(X,\|\cdot\|_{\left(\alpha_{1}\right)}\right)^{*} .
$$

Since $\left(X,\|\cdot\|_{\left(\alpha_{1}\right)}\right)^{*}$ has the AP, by Theorem 4 , we obtain

$$
\sum_{n=1}^{\infty} J\left(x_{n}\right)\left(x_{n}^{*}\right)=0
$$

so $\sum_{n=1}^{\infty} x_{n}^{*}\left(x_{n}\right)=0$, by again Theorem $4,(X,\|\cdot\|)$ has the AP.

The following proposition is the solution for the other part of the dual problems.

Proposition 3. There exists a Felbin-fuzzy normed space $(X,\|\cdot\|)$ which has the AP but $(X,\|\cdot\|)^{*}$ does not have the AP. does not have the AP.

Proof. Let us consider a Banach space $X$ having the AP whose dual fails to have the AP (see Reference [13]). We denote this Banach space by $\left(X,\|\cdot\|_{0}\right)$. Let us define

$$
\|x\|(t)= \begin{cases}1, & t=\|x\|_{0} \\ 0, & \text { otherwise }\end{cases}
$$

It is clear that $\|x\|_{\alpha}^{+}=\|x\|_{0}=\|x\|_{\alpha}^{-}$for all $\alpha \in(0,1]$. Clearly, $(X,\|\cdot\|)$ is complete fuzzy normed space and hast the AP. 
Now we claim that $\left(X,\|\cdot\|_{0}\right)^{*}=(X,\|\cdot\|) *$ as vector spaces. Let $\left.x^{*} \in X,\|\cdot\|_{0}\right)^{*}$. Then there exists a positive $M>0$ such that

$$
\left|x^{*}(x)\right| \leq M\|x\|_{0}=\|x\|_{\alpha}^{-}=\|x\|_{\alpha}^{+}, \forall \alpha \in(0,1],
$$

it follows $x^{*} \in(X,\|\cdot\|)^{*}$, i.e. $\left(X,\|\cdot\|_{0}\right)^{*} \subseteq(X,\|\cdot\|)^{*}$. The converse can be obtained the same way. Moreover, we have

$$
\left\|x^{*}\right\|_{\alpha}^{*+}=\left\|x^{*}\right\|=\left\|x^{*}\right\|_{\alpha}^{*-}, \forall \alpha \in(0,1] .
$$

Then we have that $(X,\|\cdot\|)^{*}$ does not have the AP.

\section{Conclusions and Further Works}

In this paper, we have studied approximation properties in Felbin-fuzzy normed spaces and developed topological tools to analyse such approximation properties. We have identified approximation properties in Felbin fuzzy normed spaces in terms of infinite sequences. By using this, we provided partial solutions about dual problems for approximation properties in Felbin fuzzy normed space. We hope that our approach may present topological foundations to research topological objects including compact subsets and weakly compact subsets in Felbin-fuzzy normed spaces. Moreover, dual problems for approximation properties can be answered completely.

Author Contributions: Conceptualization, K.Y.L.; Methodology, K.Y.L. and J.M.K.; Writing-original draft, K.Y.L. All authors have read and agreed to the published version of the manuscript.

Funding: The first author was supported by NRF-2018R1D1A1B07043566 funded by the Korean Government. The corresponding author was supported by NRF-2017R1C1B5017026 funded by the Korean Government.

Conflicts of Interest: The authors declare no conflicts of interest.

\section{References}

1. Katsaras, A.K. Fuzzy topological vector spaces. Fuzzy Sets Syst. 1984, 12, 143-154. [CrossRef]

2. Felbin, C. Finite dimensional fuzzy normed linear spaces. Fuzzy Sets Syst. 1992, 48, 239-248. [CrossRef]

3. Kaleva, O.; Seikkala, S. On fuzzy metric spaces. Fuzzy Sets Syst. 1984, 12, 215-229. [CrossRef]

4. Bag, T.; Samanta, S.K. Finite dimenstional fuzzy normed linear spaces. J. Fuzzy Math. 2003, 11, $687-705$.

5. Bag, T.; Samanta, S.K. A comparative study of fuzzy norms on a linear space. Fuzzy Sets Syst. 2008, 159, 685-707. [CrossRef]

6. Hasankhani, A.; Nazari, A.; Saheli, M. Some properties of fuzzy Hilbert spaces and norm of operators. Iran. J. Fuzzy Syst. 2010, 7, 129-157.

7. Binazr, T.; Peter, F.; Nadanban, S. A study of boundedness in fuzzy normed linear spaces. Symmetry 2018, 11, 923. [CrossRef]

8. Wu, H.C. Duality in fuzzy sets and dual arithmetics of fuzzy sets. Mathematics 2019, 7, 11. [CrossRef]

9. Cho, Y.J.; Rassias, T.M.; Saadati, R. Fuzzy normed spaces and fuzzy metric spaces. In Fuzzy Operator Theory in Mathematical Analysis; Springer: Cham, Switzerland, 2018; pp. 11-43.

10. Banach, S. Théorie des opérations Linéaires; Monografje Matematyczne: Warsaw, Poland, 1932.

11. Enflo, P. A counter example to the approximation problem. Acta. Math. 1973, 130, 309-317. [CrossRef]

12. Grothendieck, A. Produits tensoriels topologiques et espaces nucleires. Mem. Am. Math. Soc. 1955, 16, 1-140.

13. Lindenstrauss, J.; Tzafriri, L. The approximation property. In Classical Banach Spaces I, Sequence Spaces; Springer: Berlin, Germany, 1977; pp. 29-42.

14. Yilmaz, Y. Schauder bases and approximation property in fuzzy normed spaces. Comput. Math. Appl. 2010, 59, 1957-1964. [CrossRef]

15. Lee, K.Y. Approximation properties in fuzzy normed spaces. Fuzzy Sets Syst. 2016, 282, 115-130. [CrossRef]

16. Kim, J.M.; Lee, K.Y. Approximation properties in Felbin-fuzzy normed spaces. Mathematics 2019, 7, 1003. [CrossRef]

17. Konwar, N.; Davvaz, B.; Debnath, P. Approximation of new bounded operators in intuitionistic fuzzy nBanach spaces. J. Intell. Fuzzy Syst. 2018, 35, 6301-6312. [CrossRef] 
18. Saheli, M. A comparative study of fuzzy norms of linear operators on a fuzzy normed linear spaces. J. Math. Model. 2015, 2, 217-234.

19. Bag, T.; Samanta, S.K. Fuzzy bounded linear operators. Fuzzy Sets Syst. 2005, 151, 513-547. [CrossRef]

20. Bag, T.; Samanta, S.K. Fuzzy bounded linear operators in Felbin's type fuzzy normed linear spaces. Fuzzy Sets Syst. 2008, 159, 685-707. [CrossRef]

21. Xiao, J.; Zhu, X. On linearly topological structure and property of fuzzy normed linear space. Fuzzy Sets Syst. 2002 125, 389-399. [CrossRef]

22. Megginson, R.E. Vector topologies. In An Introduction to Banach Space Theory; Springer: New York, NY, USA, 1988; pp. 180-181.

23. Kim, J.M. Characterizations of bounded approximation properties. Taiwan. J. Math. 2008, 12, $179-190$. [CrossRef]

(C) 2020 by the authors. Licensee MDPI, Basel, Switzerland. This article is an open access article distributed under the terms and conditions of the Creative Commons Attribution (CC BY) license (http://creativecommons.org/licenses/by/4.0/). 